\title{
A Study of Erythron Status in Pregnant \& Non-Pregnant Age Matched Females of Jodhpur Region
}

\author{
Ruchi Kothari ${ }^{1}$, Pradeep Bokariya ${ }^{2}$, Varun Kothari ${ }^{3}$ \\ ${ }^{I}$ Assistant Professor, Department of Physiology, MGIMS, Sevagram \\ ${ }^{2}$ Assistant Professor, Department of Anatomy, MGIMS, Sevagram \\ ${ }^{3}$ Lecturer, Dept of Orthodontics, Vyas Dental College \& Hospital, Jodhpur
}

\begin{abstract}
:-
Objective: The effect of normal gestation on erythron status is a theme of continual interest in the developing countries like India. The present study was undertaken to evaluate and compare the haematological parameters related to the erythrocytes in pregnant and the normal age matched females of Jodhpur region.

Material \& Methods: A total of 30 healthy non-pregnant females and 30 pregnant women were examined for erythron related parameters which included total RBC count, Haemoglobin concentration, Packed cell volume, MCH (Mean Corpuscular Haemoglobin), MCHC (Mean Corpuscular Haemoglobin Concentration) \& Reticulocyte count. The subjects were of the age group 18-40 years. The Total RBC was done by haemocytometry method, $\mathrm{Hb}$. estimation by Cyan MethHb method and PCV by Wintrobe's method. The red cell indices were calculated using the standard formulae. The reticulocyte count was done using Brilliant Cresyl Blue as the stain. The corrected Reticulocyte count was also calculated for each of the subjects.

Statistical Analysis: The data thus obtained was analysed using statistical software EPI info 6.0. For validation of observations, Student's " $\mathrm{t}$ " test has been employed.

Results: The mean Total RBC count among the control subjects was found to be $4.42 \mathrm{million} / \mathrm{mm}^{3}$ whereas it was $3.88 \mathrm{million} / \mathrm{mm}^{3}$ in pregnant women. The average $\mathbf{H b}$ concentration in controls was $12.2 \mathrm{~g} / \mathrm{dl}$ and that in pregnant women was $10.2 \mathrm{~g} / \mathrm{dl}$. The value of PCV in controls \& pregnant females was $41 \%$ and $37 \%$ respectively. Similarly the MCH \& the MCHC was found out to be $27.65 \mathrm{pg} \& 29.81 \%$ respectively for the control females and $26.28 \mathrm{pg} \& 27.56 \%$ respectively for the pregnant women.

The Reticulocyte count was $1.2 \%$ in control group and $5 \%$ for the pregnant females.

Conclusion: The values of all erythron parameters in pregnant women are significantly low as compared to age matched controls, except reticulocyte count which indicates accelerated erythropoiesis to a great extent during pregnancy. This reticulocytosis was found in association with anemia and thus represents a response of haematopoietic system to physiologic stimulation of maternal erythropoeisis during pregnancy.
\end{abstract}

Keywords: erythron, pregnant, age-matched controls, reticulocyte count.

\section{INTRODUCTION}

The circulating fluid "blood" is part of a tissue of the body named the "ERYTHRON" which includes not only the circulating red blood cells and plasma but also the haemopoietic tissues of the bone marrow and extra-medullary blood forming centres [1]. The concept of erythron emphasizes the functional unity of RBCs and their pre-cursors. In "Pregnancy" which represents a period of stress and poses a major physiological challenge to the human body, a number of profound haematological changes occur in the maternal hematologic system to allow the system to adjust to the newly formed maternal-fetal unit.[2] The physiologic adjustments which the parous state necessitates provide an appropriate supply of oxygen and other nutrients to the fetus, placenta \& hypertrophied maternal tissues. These conditions in human beings are made possible by a variety of changes including alterations in the red blood cells. In the physiologically normal woman these changes take place without adverse alterations in overall body function.[3]

Thus many of the hematological standards considered normal in non-pregnant females are not appropriate during pregnancy. Conflicting results have been documented regarding the statistical significance of variations in some haematological parameters at different trimesters of pregnancy. Environmental factors, ethnic and tribal peculiarities have variously been implicated. Jodhpur being typically hot \& dry, is a part of Rajasthan which is the largest state of India situated in its western zone where the Thar Desert lies. Apart from the climatic disparities there are large differences in the cultural, social, nutritional and professional activities of the people here from that of the western countries. This is reflected in their feeding habits and dietary intake of nutrients in varying proportions. Local food taboos for pregnant women are also many \& are commonly adhered to during most of the pregnancy. In the course of pregnancy, iron absorption is often inhibited by high phytate content of many of the grain based diets in the tropics [4] All these facts suggest that it is almost undesirable to accept and 
follow the haematological values and ranges established on the basis of foreign studies; for the purpose of this part of the country as these may not be completely appropriate owing to well recognised nutritional, environmental and genetic factors, which influence them.

Hence it necessitated undertaking the present survey on status of erythron in this population and analysing the impact of pregnancy on the same. The comparisons between the observations of the various red cell parameters including the reticulocyte count constituting the erythron status of an individual in females of different age groups of the present study are made with the values reported by different researchers all over the world. It was conducted as a pilot study incorporating only a few representative samples for estimation of red cell parameters in normal pregnancy \& their comparison with age matched controls.

\section{MATERIAL \& METHODS}

The present study was carried out on 50 expectant (pregnant) and 50 healthy non pregnant females of Jodhpur region and the range of age groups was from 18-40 years. The blood specimens were collected for the study from subjects, all of whom were clinically healthy that satisfied the inclusion and exclusion criteria and were taking no medication. The exclusion criteria included fever in the past week, a history or features suggestive of haemoglobinopathy, chronic medical ailments, bleeding in early pregnancy and other major complications of pregnancy (e.g. hypertension, diabetes, and pre-eclampsia/eclampsia). All females with a known disability likely to have bearing or influence on the erythron status were excluded from the survey. The young non pregnant women in this series were mainly females who came for their routine check ups, counselling for menstrual irregularities excluding menorrhagia or premarital examinations. The selected females were asked to answer a self coding questionnaire. Besides these, a group of 50 expectant (pregnant) ladies of the same age group that were apparently healthy were examined during the last trimester of pregnancy when they reported for the fortnightly check up in the antenatal clinic of Government Hospital of Jodhpur. Their particulars regarding age, obstetric history, dietetic habits including the intake of folate and iron, past illness, general health, structure of household whether housebound, catering arrangements, socio economic status of family etc. were entered in a proforma and were investigated for blood group also in addition to the parameters under study. An informed consent was taken prior to the investigations by each of the subjects. The study was approved by Institutional Ethics committee. All estimates were done on venous blood. The venous blood for all samples collected for the study was drawn between 8:30 a.m. to 12 noon and following estimations were done-

a) Total R.B.C. Count

This was done by Hemocytometry method using an Improved Neubauer's Double counting chamber.

b) Haemoglobin- It was done by "Cyanmeth Hb. Estimation Method"

c) Packed Cell Volume - It was done by "Wintrobe's (Macro) Method"

d) Red Blood Cell Indices-

Mean Corpucular Haemoglobin (M.C.H.) $=\underline{\mathrm{Hb} . \text { in } \mathrm{g} / 100 \mathrm{ml} \mathrm{X} 10}$

R.B.C.count (millions/cumm.)

Mean Corpuscular Haemoglobin Concentration (M.C.H.C.) $=$ Hb. in $\mathrm{g} / \mathrm{dl}$ X 100

P.C.V. per $100 \mathrm{ml}$ blood

e) Total Reticulocyte Count - It was done using Brilliant Cresyl blue technique and was calculated by the formula-

Reticulocyte $(\%)=$ [Number of Reticulocytes / Number of Red Blood Cells $]$ X 100

In order to get a more accurate assessment of bone marrow function, the calculated reticulocyte percentage (\%) was corrected with a calculation called a corrected reticulocyte count or a reticulocyte index (RI). This calculation compares the patient's hematocrit with a normal hematocrit value. An additional calculation called the reticulocyte production index (RPI) is was also calculated to correct for the degree of reticulocyte immaturity - reflecting how quickly the reticulocytes were released from the bone marrow and how long it will take them to mature in the bloodstream.

Reticulocyte Index = Reticulocyte count (\%) X [Measured hematocrit / Normal hematocrit]

Reticulocyte Production Index $=($ Reticulocyte Index $) X(1 /$ maturation time $)$

\section{Statistical Analysis}

The data thus obtained was analysed using statistical software EPI info 6.0. For all hematologic variables parametric analysis using mean, Standard deviation (S.D.), Standard Error (S.E.) was done. For validation of observations, Student's " $\mathrm{t}$ " test has been employed to compare two mean values. All tests were at the 5\% statistical level $(\mathrm{P}<0.05)$

\section{RESULTS}


Mean RBC count in the group of pregnant ladies as shown in the above table came out to be $3.88 \pm$ 0.55 millions/ cubic $\mathrm{mm}$ whereas in non pregnant females it was $4.42 \pm 0.54$. millions/ cubic mm. Mean $\mathrm{Hb}$ concentration in gravid females was estimated to be $10.2 \pm 1.13 \mathrm{~g} / \mathrm{dl}$ of blood whereas the same for non-gravid was $12.2 \pm 1.59 \mathrm{~g} / \mathrm{dl}$ of blood. The mean packed cell volume for both groups i.e. pregnant and non pregnant was determined as $37 \pm 5.35$ and $41 \pm 4.89$ respectively. The average $\mathrm{MCH}$ and MCHC values as calculated using standard formulae were $26.28 \pm 1.81 \mathrm{pg} \& 27.56 \pm 3.16 \%$ respectively for pregnant women \& those for the nonpregnant ones were $27.65 \pm 3.84$ and $29.81 \pm 3.13 \%$ respectively. The mean reticulocyte count was $5 \pm 0.28 \% \&$ $0.4-1.4 \%$ for the two groups.

\section{DISCUSSION}

The maintenance of normal blood counts during gestation is very relevant in the overall wellbeing of a woman. During normal pregnancy, characteristic changes are observed in the Hb conc. and in the erythrocyte indices. These changes have been attributed mainly to the physiologic haemodilution in the past [5].

\section{Total RBC Count}

An earlier study was undertaken in Nigeria where the pregnant ladies were divided into three groups on the basis of trimester of pregnancy and their haematological indices were compared with those of non-pregnant age matched women volunteers as controls.[6] Using the WHO minimum acceptable standard [7], almost 3 quarters $(73.3 \%)$ of their pregnant population presented with a mild or moderate form of anaemia but only $1.2 \%$ with severe anaemia. Their mean figure of total RBC count was far less than the age-matched control value at Jodhpur. One of the factors which is responsible for this relatively low figure has been hypothesized on the basis of specific tailored investigations [8] as that inspite of pregnancy related enhanced erythropoiesis, the increase in plasma volume of the greater magnitude renders total count to these lower mean values despite expansion in total red-cell volume. The physiologic necessity for RBC increase is obvious when one considers the needs of developing fetus and the needs of the mother in relation to the increased oxygen requirements and the blood loss occurring at delivery [2] however isolated analysis of data of total RBC count for both the groups of females in our study; manifests a pattern of significant reduction in RBC Count during pregnancy.

\section{Haemoglobin}

The occurrence of anemia in pregnancy has been reported in different parts of the world with an incidence up to 56\%. [9] Its effects are more marked in developing countries like India, due to lack of balanced dietary intake, short inter-pregnancy interval and prolonged lactation, coupled with women's daily routine of heavy physical exercise. The increased requirements of pregnancy and inadequate intake; stemming from anorexia, nausea, vomiting and lack of economic access to appropriate foods have far reaching effects on the mother and fetus.

Over $90 \%$ of anaemia worldwide is due to iron deficiency associated with depleted iron stores and deficient intake. As pregnancy advances, serum iron falls and total iron binding capacity (TIBC) increases due to plasma volume. Other potential causes of anaemia include malaria, hookworm infestation, placental hormones secreted in pregnancy [6]. According to Pritchard, anemia during pregnancy occurs if there is a $\mathrm{Hb}$ concentration of $<10 \mathrm{~g} / \mathrm{dl}$. In clinical studies, several authors have used discriminatory Hb levels in pregnancy ranging from $10-11.4 \mathrm{~g} / \mathrm{dl}[10,11,12]$. The $5^{\text {th }}$ percentile value of $\mathrm{Hb}$ in 107 placebo treated pregnant women at third trimester has been reported as $10.0 \mathrm{~g} / \mathrm{dl}$. [13] In Nigeria reported $\mathrm{Hb}$ value in the pregnant women at third trimester is $9.80 \mathrm{~g} / \mathrm{dl}$ (9.61-10.00) while in non-pregnant females it was reported as $12.05 \mathrm{~g} / \mathrm{dl}$ (11.66-12.44) [6]. The observations on the haemoglobin estimation in the present study are comparable to those quoted in the available literature. For the low value of mean $\mathrm{Hb}$. found in pregnant ladies, hydraemia of pregnancy could be ascertained as one of the possible cause. This physiologic anemia of pregnancy is thought to be a dilutional process secondary to an increase in plasma volume. In normal pregnancy, plasma volume increases from $25 \%$ to $80 \%$ Using RBCs labeled with $\mathrm{Cr}^{51}$, blood volumes in normal pregnant females have been calculated and showed to have an average increase of $48 \%$ in females with singleton pregnancies. [8]

While another probable contributory factor for low $\mathrm{Hb}$. values could be the "diet" of these women as iron deficiency is common in females of child-bearing years in this local specific region. Many women of childbearing age have iron-deficiency and reduced $\mathrm{Hb}$. with low RBC count. Pregnant women with anaemia are at an increased risk of miscarriage, stillbirths, premature birth, delivery of babies with intrauterine growth retardation and low birth weight, who are also prone to infection because of reduce immune competence. [6]

\section{Packed Cell Volume/ Haematocrit}

The pattern of variation of packed cell volume in females of this region is similar to that of the reported values [8] upto a considerable range. But the pattern of PCV exhibits a sharp contrast in between females of 
both the groups as is shown in the table. Peculiarly there appears a nadir in gravid females as compared to non gravid ones.

The values of some important haematological parameters were also determined in 130 healthy pregnant subjects between 18-43 years in Nigeria where subjects were divided into three groups according to three trimesters [14]. The results obtained in their study indicated that Haematocrit (Hct) showed a significant difference i.e. it was highest amongst subjects in the third trimester. This is not in accordance to the observation of the present study. As regards this discrepancy of present values is concerned, possibly it indicates relatively more increase in the plasma volume although the extent needs to be confirmed through appropriate studies on total red cell mass as well as total plasma mass for local population so as to ascertain any difference in quantum or magnitude of plasma expansion vis-a-vis red cell mass increase for comparison with the reported values.

\section{MCH \& MCHC}

The $\mathrm{MCH}$ indicates the mean content of $\mathrm{Hb}$ in the population of erythrocytes. Values of $<27 \mathrm{pg}$ suggest iron deficient erythropoeisis in non-pregnant women. [15] The MCHC is a mathematical calculation dependent on haematocrit \& $\mathrm{Hb}$. It denotes $\mathrm{Hb}$ content in the erythrocyte mass being expressed as $\mathrm{g} \mathrm{Hb}$ per $\mathrm{dl}$ erythrocytes. The utility of erythrocytes indices including MCHC depends on quality of analysis. It is also stated that MCHC is of no use during pregnancy due to great interindividual variation in the degree of haemodilution [16] In healthy non pregnant women $\mathrm{MCHC}$ value $<30 \mathrm{~g} / \mathrm{dl}$ suggests iron deficient anemia.

The mean values of M.C.H. and M.C.H.C. in the present study were found to be lower in pregnant females. This is although not in consonance with the available literature but the values obtained were well within the normal limits.

\section{Reticulocyte Count}

The reticulocyte count in peripheral blood is a measure of demand in response of erythropoeisis and the level of circulating reticulocytes continues to be considered a reliable index of effective erythropoeisis. The picture of reticulocyte count projects a reverse pattern in comparison to RBC count in pregnant ladies. The control of RBC production during pregnancy is rather complex. Erythropoietin which stimulates erythrocyte production in the bone marrow, is increased during pregnancy [17].The red cell mass (RCM) also increases during pregnancy about 20-30\% above normal [18]. This increase is obviously elicited by increased production of erythropoietin which acts as a physiologic stimulator of maternal erythropoeisis during pregnancy. Increase in maternal erythropoeisis during $3^{\text {rd }}$ trimester is found to be preceded by increase in serum erythropoeitin concentration presumably on basis of decreased oxygen delivery to maternal renal tissues during late $2^{\text {nd }}$ and early $3^{\text {rd }}$ trimester [13]. Human placental lactogen augments the action of Ep but this effect is inhibited by estrogen, which also inhibits erythropoietin directly. Progesterone negates these effects of estrogen such that the overall effect of the interaction of these hormones is a positive one.[19] As a result, the bone marrow is hyperplastic and there is a slight increase in the reticulocyte count.[17] "Reticulocytosis" noted in pregnancy is associated with anemia and thus represents a response of haematopoietic system. The significance of reticulocytosis is in association with demands upon the haematopoeitic system. If stimulus to accelerated red cell production is sufficiently great, immature cells are liberated from bone marrow.

During the latter half of pregnancy, the red cell production is increased and the lifespan of newly formed cells is unaltered as was postulated in a study in the near term pregnant subjects, where the reticulocyte count \& red cell glycolytic activity were significantly elevated $(\mathrm{p}<0.01)$ above the values found in normal nonpregnant women. There was a small but significant reticulocytosis (Reticulocyte Count-1.80\%) detected in the pregnant subjects as compared to the value of reticulocyte count (1.06\%) in non pregnant subjects.[8] This conclusion of increased erythropoeitic activity manifested by an increase in the mean percentage of reticulocytes had been bolstered by increased red cell glycolysis in that study. The young red cells, primarily those that are reticulated metabolize glucose much faster than do the older erythrocytes. Therefore the elevated erythrocyte glycolytic activity may serve as an indication of the recent addition of new red cells to the peripheral circulation [20] These observations in the pregnant women point to an increased production of red cells although low RBC counts are encountered during pregnancy. This corroborates well with the findings of the present study as our measurements during the latter half of pregnancy; of reticulocyte count also indicate that normally at this time there is accelerated erythropoeisis although the 5 times reticulocyte count in pregnancy is not in accordance with previous studies.

\section{CONCLUSION}

The present study shows that in this geospecific region of typically hot environment $\&$ dry climate, all the erythrocyte indices values in third trimester pregnant ladies were significantly low as compared to age matched controls, except reticulocyte count which was found to be 5 times the value of controls indicating accelerated erythropoiesis to a great extent. Abnormalities of erythron status, though usually with mild clinical 
presentation could be an alarm and we suggest pre-conception care should be formulated into primary healthcare to serve women in the childbearing age group.

\section{REFERENCES}

[1]. Witts CL. The Pathology and Treatment of Anemia. Lancet 1932; 1: 495

[2]. Peck TM, Arias F. Hematologic changes associated with pregnancy. Clin Obstet Gynec 1979; 22(4): 785-98

[3]. Bell WR. Hematologic Abnormalities in Pregnancy. Med Clin North Am 1977; 61(1): 165-99

[4]. Reviews April. Anaemia in pregnancy in development countries. Brit J Obstet Gynaec 1998: 05; 385 90.

[5]. Taylor DJ, Lind T. Haematological changes during normal pregnancy: iron induced macrocytosis. $\mathrm{Br} \mathbf{J}$ Obstet Gynaecol 1976; 83:760-7.

[6]. Akingbola TS, Adewole IF, Adesina OA et al. Haematological profile of healthy pregnant women in Ibadan, south-western Nigeria. J Obstet Gynaecol 2006; 26:763-9.

[7]. WHO. Nutritional anaemias. Technical Report Series 503.Geneva: 1972.

[8]. Pritchard JA, Adams RH. Erythrocyte production and destruction during pregnancy. Amer J Obstet Gynec 1960; 79: 750-57

[9]. World Health Organization. The prevalence of anemia in women: a tabulation of available information. $2^{\text {nd }}$ edition, Geneva: World Health Organization, Maternal Health and Safe Motherhood Programme, Nutrition Programme WHO/MCH/MSM/92.2, 1992

[10]. Koller O, Haram K, Sagen N. Maternal hemoglobin concentration and fetal health. In. Bern M.M.M, Frigoletto F.D.eds. Hematologic disorders in maternal-fetal medicine. New York, Wiley-Liss 1989; 3146

[11]. Milman N, Agger OA, Neilsen OJ. Iron supplementation during pregnancy. Effect on iron status markers, serum erythropoeitin and human placental lactogen. A placebo controlled study in 207 Danish women. Dan Med Bull 1991; 38: 471-6

[12]. Eskeland E, Malterud K, Ulvik RJ, Hunsakaar S. Iron supplementation in pregnancy is less enough? Acta Obstet Gynec Scand 1997;76:822-8

[13]. Milman N, Byg KE, Agger AO. Hemoglobin and erythrocyte indices during normal pregnancy and postpartum in 206 women with and without supplementation. Acta Obstet Gynec Scand 2000; 79 : 8998

[14]. Dapper DV, Ibe CJ, Nwauche CA. Haematological values in pregnant women in Port Harcourt, Nigeria. Niger J Med 2006; 15(3):237-40.

[15]. Roberts WE, Morrison JC. Evaluation of anemia in pregnancy. In: Kitay D.Z., ed. Hematologic problems in pregnancy. Oradell, Medical Economics Books 1987; 15-26

[16]. Cabaniss CD, Cabaniss ML. Physiologic hematology of pregnancy. In: Kitay D.Z., ed Hematologic problems in pregnancy. Oradell, Medical Economics Books 1987; 3-14

[17]. Jepson JH. Endocrine control of maternal and fetal erythropoeisis. Can Med Assoc J 1968; 98 : 844

[18]. Letsky EA. Blood volume, haematinics, anaemia. In: de Swiet M, editor. Medical disorders in obstetrics practice. Oxford: Blackwell Science.1995. pp $33-70$

[19]. McFee JG. Anemia in pregnancy: A reappraisal. Obstet Gynecol Surv 1973; $28: 769$

[20]. Hollingsworth JW. Erythrocyte glycolysis in hemolytic disease. J Lab Clin Med 1955; 45: 920

Table No.1 Erythron Status of Pregnant \& Control Females

\begin{tabular}{|c|c|c|c|c|c|c|}
\hline & & Pregnant $(n=$ & & Controls (n=5 & & \\
\hline S.No & Erythron Status & Mean Values & Range & Mean Values & Range & P Value \\
\hline 1. & $\begin{array}{l}\text { Total RBC Count } \\
\left(\mathrm{million} / \mathrm{mm}^{3}\right)\end{array}$ & $3.88 \pm 0.55$ & $3.13-4.95$ & $4.42 \pm 0.54$ & $3.53-6.22$ & $<0.05$ \\
\hline 2. & $\mathbf{H b}(\mathrm{g} / \mathrm{dl})$ & $10.2 \pm 1.13$ & $9-13$ & $12.2 \pm 1.59$ & $8.0-14.2$ & $<0.05$ \\
\hline 3. & PCV (\%) & $37 \pm 5.35$ & $30-48$ & $41 \pm 4.89$ & $32-50$ & $<0.05$ \\
\hline 4. & MCH (pg) & $26.28 \pm 1.81$ & $23.02-30.47$ & $27.65 \pm 3.84$ & $20.67-33.25$ & $>0.05$ \\
\hline 5. & MCHC (\%) & $27.56 \pm 3.16$ & $24.28-37.57$ & $29.81 \pm 3.13$ & $25-36.21$ & $>0.05$ \\
\hline 6. & $\begin{array}{l}\text { Reticulocyte Count } \\
(\%)\end{array}$ & $5 \pm 0.28$ & $0.4-1.4$ & $1.2 \pm 0.37$ & $0.4-2.0$ & $<0.05$ \\
\hline
\end{tabular}

